\title{
Preparation and characterization of nanomaterial of $\mathrm{LaAl}_{11} \mathrm{O}_{188}$ produced by chemical co-precipitation method
}

\author{
Zhao Tonghui ${ }^{12)} \quad$ Zhou Zhen $^{1)}$ Lv Tianling ${ }^{2)}$ Zhao Rui ${ }^{2)}$ \\ 1)( School of Measurement and Communication, Harbin University of Science \& Technology \\ Harbin 150080) \\ 2)( Labor Safety Science and Technology Research Center of Heilongjiang Province Harbin \\ 150001)
}

Key words: Chemical co-precipitation method; Co-precipitation- hydro-thermal method; Nano-materials

\begin{abstract}
In this paper the methods of chemical co-precipitation method were used to produce nanomaterial of $\mathrm{LaAl}_{11} \mathrm{O}_{18}$. In order to find the effects of the preparation parameters on its micoscopic structure and the influences of calcinated temperature, keeping warm time and $\mathrm{pH}$ value on organizational structure and generate efficiency. And use the X-ray powder diffraction (XRD) and the scanning electron microscopy (SEM) to find the impacts of calcinated temperature , keeping warm time and $\mathrm{pH}$ value on the composition and micoscopic structure.
\end{abstract}

\section{Preface}

$\mathrm{LaAl}_{11} \mathrm{O}_{18}$ composite oxide, as a new inorganic functional material, has been widely used in many areas of research since 70s. For instance, it can be used as laser material and the crystal matrix of superconducting and fluorescent materia. As the catalytic materials of high temperature catalytic combustion reaction,particularly, it has been wildly researched ${ }^{[1-3]}$. $\mathrm{LaAl}_{11} \mathrm{O}_{18}$, a new thermal barrier coating materials, is a good alternatives of the zirconia which is partially stable to Yttrium oxide. $\mathrm{LaAl}_{11} \mathrm{O}_{18}$ is magnetic lead iron ore structure which is a mixed structure of cube and hexagonal body. In this structure, One layer of $\mathrm{LaO}_{3}$ which is found showing crystalline mirror next to four layers of aluminum spinel layerwhich is divided by symmetrical mirror. In the layer of LaO3, Highly loaded $\mathrm{La}^{3+}$ is located at the oxygen position in the hexagonal tight structure of the oxygen ions. $\mathrm{La}^{3+}$ diffusion is strongly suppressed so that the growth rate of the layered structure compound in the C-axis direction perpendicular to the mirror surface is relatively slow, which makes it has good thermal stability. Compared to the oxygen ion conductor of zirconia, the applicable temperature of $\mathrm{LaAl}_{11} \mathrm{O}_{18}$ is allowed at $130{ }^{\circ} \mathrm{C}$ for the thermal stability of $\mathrm{LaAl}_{11} \mathrm{O}_{18}$. In addition, this arrangement makes the holes in the structure very little, which can effectively inhibit the diffusion of oxygen atoms, reduce the bonding layer and the substrate oxidation rate, reduce the amount of oxide, thereby improving the coating reliability and service life $\mathrm{e}^{[3-6]}$.

\section{Preparation of test materials}

In the coprecipitation method, $\mathrm{Al}$ (NO3) 3 reacts with ammonia to produce $\mathrm{Al}(\mathrm{OH}) 3$, $\mathrm{La}$ (NO3) 3 and ammonia react to produce $\mathrm{La}(\mathrm{OH}) 3$. And, it is most important that the following reaction occurs during the heating process ${ }^{[7]}$ :

$$
2 \mathrm{Al}(\mathrm{OH})_{3} \rightarrow \mathrm{Al}_{2} \mathrm{O}_{3}+3 \mathrm{H}_{2} \mathrm{O}
$$

$$
\begin{aligned}
& 2 \mathrm{La}(\mathrm{OH})_{3} \rightarrow \mathrm{La}_{2} \mathrm{O}_{3}+3 \mathrm{H}_{2} \mathrm{O} \\
& \mathrm{La}_{2} \mathrm{O}_{3}+\mathrm{Al}_{2} \mathrm{O}_{3} \rightarrow 2 \mathrm{LaAlO}_{3} \\
& \mathrm{LaAlO}_{3}+5 \mathrm{Al}_{2} \mathrm{O}_{3} \rightarrow \mathrm{LaAl}_{11} \mathrm{O}_{18}
\end{aligned}
$$

Specific ingredients shown in Table 1. 
TABLE 1 Basic ingredients list of coprecipitation method

\begin{tabular}{cccc}
\hline Drugs & Mass (g) & Concentration (mol / L) & Volume (mL) \\
\hline $\mathrm{LaNO}_{3}$ & 1.121 & - & - \\
$\mathrm{HNO}_{3}$ & - & 15 & 30 \\
$\mathrm{AlNO}_{3}$ & 8.083 & - & - \\
$\mathrm{C}_{18} \mathrm{H}_{29} \mathrm{NaO}_{3} \mathrm{~S}$ & 0.2 & - & - \\
$\mathrm{NH}_{3} \cdot \mathrm{H}_{2} \mathrm{O}$ & - & 20 & - \\
\hline
\end{tabular}

The coprecipitation preparation step:

(1) A solution of $0.4 \mathrm{~mol} / \mathrm{L}$ and $0.2 \mathrm{~mol} / \mathrm{L}$ was prepared by dissolving $8.083 \mathrm{~g} \mathrm{Al}(\mathrm{NO})_{3} \cdot 6 \mathrm{H}_{2} 0$ and $1.121 \mathrm{~g} \mathrm{La}(\mathrm{NO})_{3} \bullet 9 \mathrm{H}_{2} \mathrm{O}$, respectively.

(2) Mix the two solutions on a stirrer and add $0.2 \mathrm{~g}$ of sodium dodecylbenzenesulfonate as a dispersant ${ }^{[8]}$.

The effect of $\mathrm{C}_{18} \mathrm{H}_{29} \mathrm{NaO}_{3} \mathrm{~S}$ :

(1)Adsorbed on the surface of the solid particles so that the surface of the agglomerated solid particles is easy to wet.

(2)making the surface of the solid particles to form a bilayer structure. The polar end of the outer dispersant has a strong affinity for water and increases the degree of wetting of the solid particles. Solid particles are separated by electrostatic repulsion.

(3)Making the system uniform, suspended performance increases and do not precipitate, so that the physical and chemical properties of the whole system. In summary, the use of dispersants can safely disperse the solid particles in the liquid. The formation of a stable dispersion system, in addition to the use of electrostatic repulsion, that is, the negative charge adsorbed on the surface of the particles mutually exclusive to prevent the particles and particles between the adsorption / aggregation and finally the formation of large particles and stratification / settlement, but also the use of The theory of the steric hindrance effect, that is, in the adsorption of negatively charged particles close to each other, so that they slide each other staggered, such as the role of steric hindrance of surfactants are generally non-ionic surfactants. Flexible use of electrostatic repulsion with the theory of steric hindrance, can constitute a highly stable dispersion system.

(3) $\mathrm{NH}_{3} \cdot \mathrm{H}_{2} \mathrm{O}$ was added dropwise to the solution to react, resulting in a white precipitate.

(4) Use $\mathrm{HNO}_{3}$ and $\mathrm{NH}_{3} \cdot \mathrm{H}_{2} \mathrm{O}$ to adjust the $\mathrm{pH}$ repeatedly to control it between 8 and 10.

(5)curing the reaction solution .

(6) After curing the solution into the hydrothermal reactor heated to 150 degrees insulation for ten hours. After the insulation naturally dropped to room temperature. After completion of the reaction, opening the reaction kettle, where it became a supernatant and the lower layer was a white precipitate.

(7) Precipitated by centrifugal.

(8) Washing and precipiting the precursors repeatly 5 times before into the insulation drying oven for 2 hours.

(9) According to the above operations, respectively, making the same group of experiments, observe the phenomenon and record.

\section{Results and discussion}

3.1Influence of calcinated temperature

As shown in Figure $1 \mathrm{a}$ ), when the calcination temperature is $1250{ }^{\circ} \mathrm{C}$, the peak intensity of the 
diffraction powder is relatively small and the peak intensity is more balanced and only a small amount of lanthanum aluminate and a small amount of lanthanum hexaerythrite produced, the content is very small. At this point the powder is mostly alumina, lanthanum oxide, aluminum hydroxide, lanthanum hydroxide and some other impurities. And the strongest peak is lanthanum aluminate, which presumably at $1250^{\circ} \mathrm{C}$ may be just the reaction temperature. And Figure $1 \mathrm{~b}$ ) significantly increased the strong peak whose intensity was significantly enhanced and less peak. And from the XRD diffraction diagram, at this time, the resulting substances are mostly $\mathrm{LaAlO}_{3}$ and $\mathrm{LaAl}_{11} \mathrm{O}_{18}$, indicating that the reaction between the oxides has begun to strengthen. By the above $\mathrm{X}$-ray diffraction analysis, it can be seen that the actual onset temperature of the $\mathrm{LaAl}_{11} \mathrm{O}_{18}$ phase is between 1200 and $1250^{\circ} \mathrm{C}$. When the holding temperature is higher than this temperature, the generation efficiency of the $\mathrm{LaAl}_{11} \mathrm{O}_{18}$ phase increases with the temperature at different temperatures.
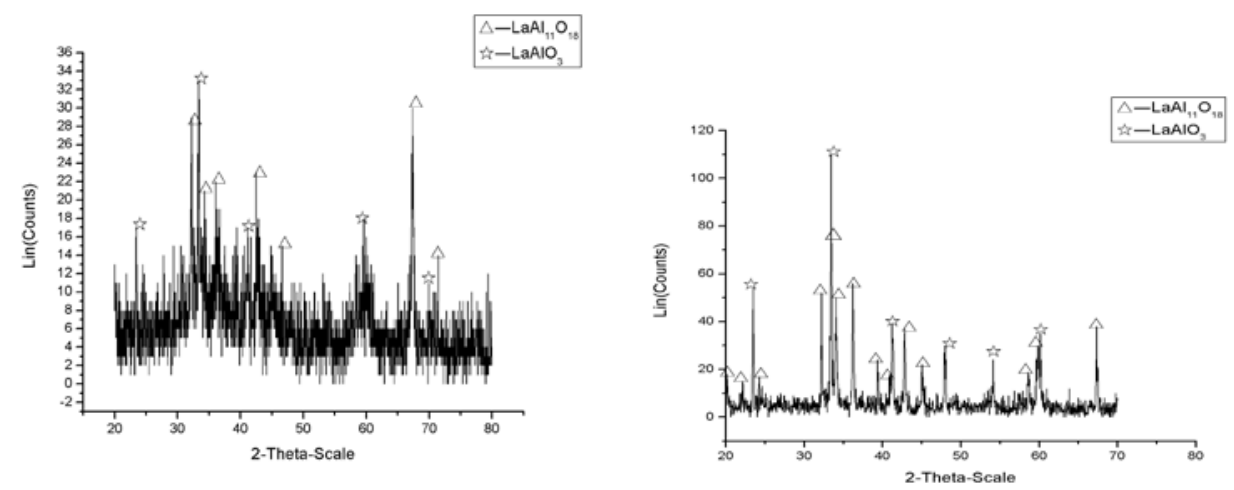

a) $1250{ }^{\circ} \mathrm{C}$ holding $2.5 \mathrm{~h} \mathrm{pH9}$

b) $1400{ }^{\circ} \mathrm{C}$ holding $2.5 \mathrm{~h} \mathrm{pH} 9$

Fig.1 X-ray diffraction pattern of powder at different heat treatment temperatures

\subsection{Influence of $\mathrm{pH}$ value}

Figure $2 \mathrm{a}$ ), b), c) three XRD test results corresponding to the samples are roasting to $1400{ }^{\circ} \mathrm{C}$, holding 4 hours under the conditions of the obtained, Wherein the $\mathrm{pH}$ of a) is 8 , the $\mathrm{pH}$ of $\mathrm{b}$ ) is 9 , and the $\mathrm{pH}$ of $\mathrm{c}$ ) is 10 . The diffraction peaks of the three samples were all obvious, and the peaks were also less and the main substances are $\mathrm{LaAlO}_{3}$ and $\mathrm{LaAl}_{11} \mathrm{O}_{18}$. This confirms the higher the sintering temperature, the longer the holding time, the more complete the reaction of this conclusion. The difference between the three is $\mathrm{pH}$. In the X-ray powder diffraction pattern, the strongest peak in Fig. 2 (b) corresponds to $\mathrm{LaAlO}_{3}$ and $\mathrm{LaAl}_{11} \mathrm{O}_{18}$. And Figure 2 a) and Figure 2 c) correspond only to a product of $\mathrm{LaAl}_{11} \mathrm{O}_{18}$. This indicates that $\mathrm{pH} 9$ is relatively easy to produce $\mathrm{LaAl}_{11} \mathrm{O}_{18}$ phase. And then from the intensity of the diffraction peak, The intensity of the diffraction peak in Fig. 2b) is significantly lower than that in Fig. 2 (a) and Fig. 2 (c). It can also be seen from Figure 3 that the sample in Figure $3 \mathrm{~b}$ ) has the highest transparency. This indicates that when the $\mathrm{pH}$ is 9, the lanthanum aluminate phase is further converted to the $\mathrm{LaAl}_{11} \mathrm{O}_{18}$ phase. It can be seen from Fig. 2 (b) that the $\mathrm{LaAl}_{11} \mathrm{O}_{18}$. phase occupies the dominant position. This indicates that the choice of $\mathrm{pH}$ is 
very important when other conditions are the same.

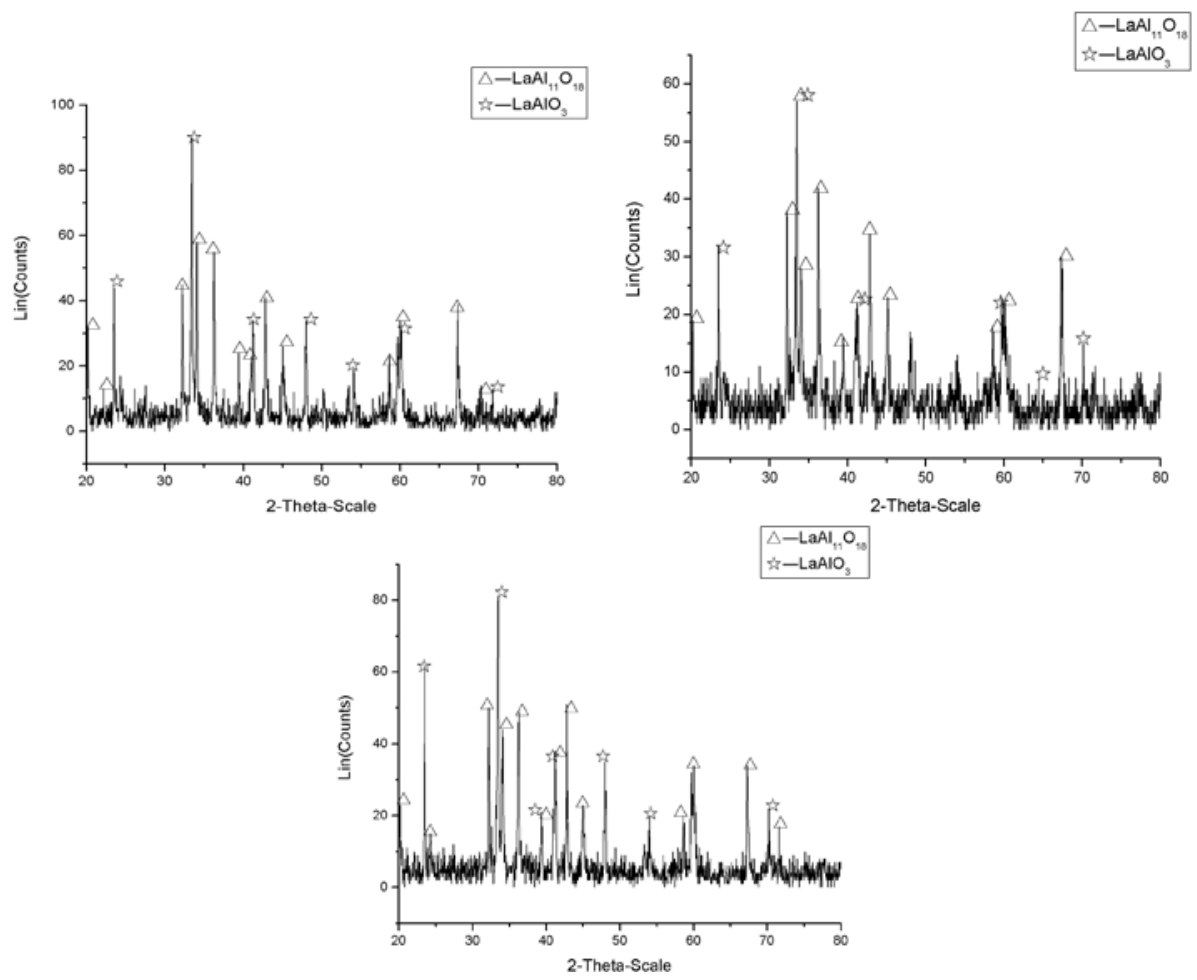
a) $1400{ }^{\circ} \mathrm{C}$ holding $4 \mathrm{~h} \quad \mathrm{pH} 8$
b) $1400{ }^{\circ} \mathrm{C}$ holding $4 \mathrm{~h} \quad \mathrm{pH} 9$
c) $1400{ }^{\circ} \mathrm{C}$ holding $4 \mathrm{~h} \quad \mathrm{pH} 10$

Figure $2 \mathrm{X}$-ray diffraction pattern of powders with different $\mathrm{pH}$

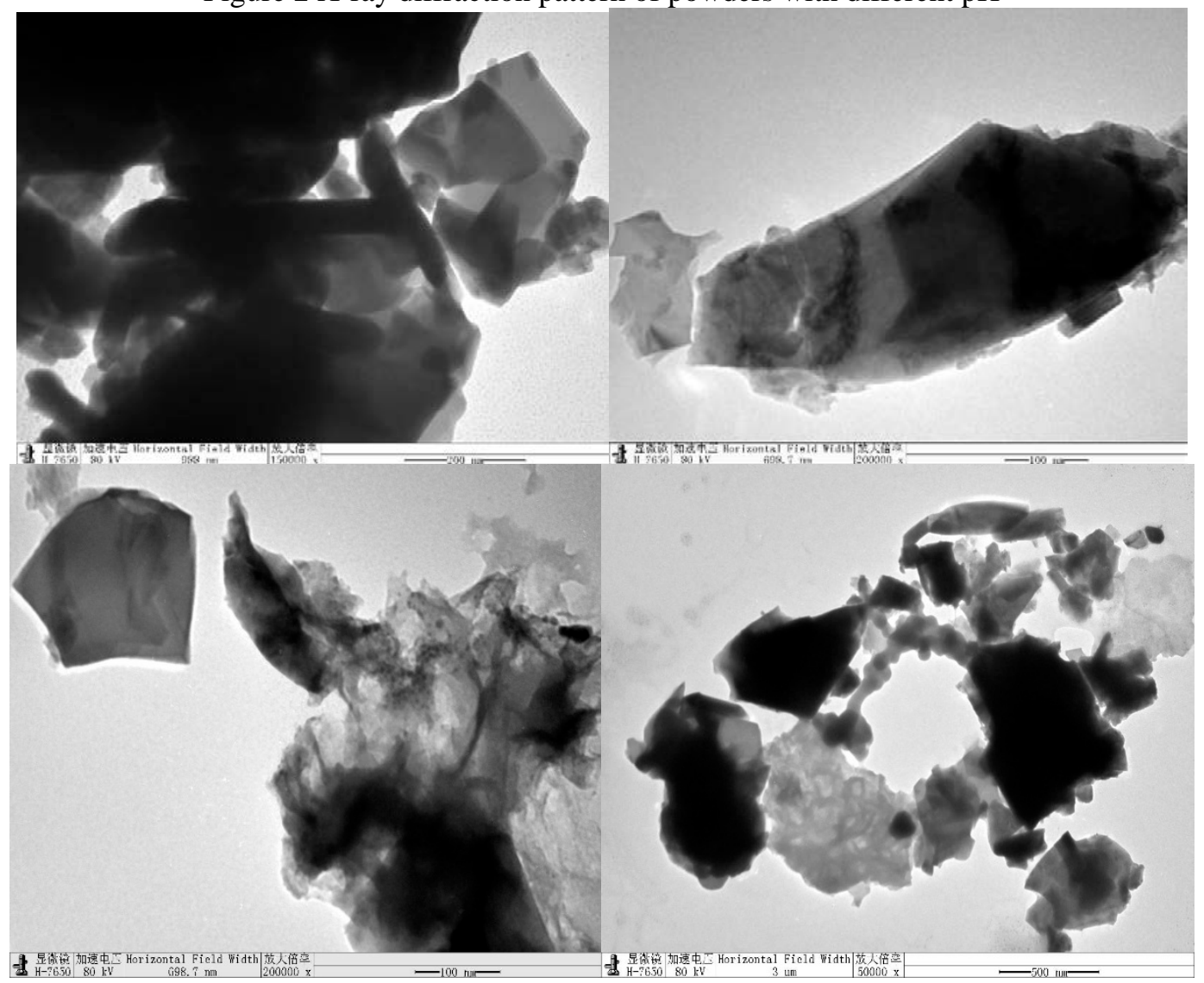




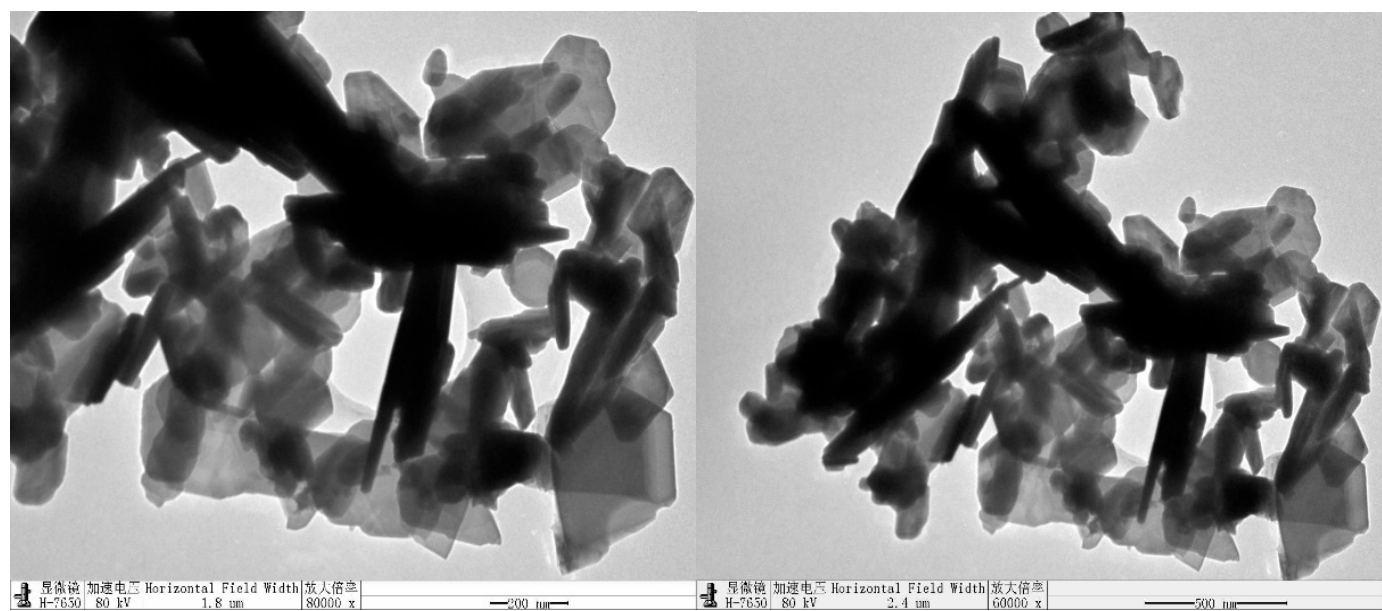

a) $1400{ }^{\circ} \mathrm{C}$ holding $4 \mathrm{~h} \quad \mathrm{pH} 8$

b) $1400{ }^{\circ} \mathrm{C}$ holding $4 \mathrm{~h} \quad \mathrm{pH} 9$

c) $1400{ }^{\circ} \mathrm{C}$ holding $4 \mathrm{~h} \quad \mathrm{pH} 10$

\subsection{Analysis of SEM}

Figure 3 Powder transmission electron microscopy at different $\mathrm{pH}$ values

In Figure 4, most of the SEM images are lamellar, with only a few small, irregularly granular, which is enough to conclude that the structure of lanthanum hexaaluminate is lamellar. And the scanning electron microscopy in the granular, clumps of things may be the $\mathrm{La}_{2} \mathrm{O}_{3}, \mathrm{Al}_{2} \mathrm{O}_{3}$ and so on which did not react completely. The front has been analyzed by XRD and transmission electron microscopy. From Fig. 4 (a), it can be seen that the thickness of the lamellae is $40 \mathrm{~nm}$ and the thickest is $120 \mathrm{~nm}$.

\section{Conclusion}

This article mainly discusses the influence of calcinated temperature , keeping warm time and $\mathrm{pH}$ value on micro structure and character during the production of the $\mathrm{LaAl}_{11} \mathrm{O}_{18}$. Conclusion as below.

(1)The key of the production of the $\mathrm{LaAl}_{11} \mathrm{O}_{18}$ is the calcinated temperature , keeping warm time. The actual generate temperature of the $\mathrm{LaAl}_{11} \mathrm{O}_{18}$ is between 1200 and 1250 degrees. When the temputer higher than this point, the production amount of the $\mathrm{LaAl}_{11} \mathrm{O}_{18}$ will increase with the calcinated temperature and keeping warm time increasing. In different temputers, the production effect of the $\mathrm{LaAl}_{11} \mathrm{O}_{18}$ will increase the calcinated temperature increasing.

(2)During the process of the $\mathrm{LaAl}_{11} \mathrm{O}_{18}$ produced by chemical co-precipitation method, $\mathrm{PH}$ has the effect on $\mathrm{LaAl}_{11} \mathrm{O}_{18} \mathrm{LaAl11O} 18$ producation efficiency. It is the highest producation efficiency and the more completely reaction of LaAl11O18 when the other experimental conditions are the same.

(3)This paper use the scanning electron microscopy (SEM) method found the Morphology of $\mathrm{LaAl}_{11} \mathrm{O}_{18}$ is lamellar and the thickness of the film is $40 \mathrm{~nm}$. 


\section{References}

[1] Qinghua Cheng, Qian Yu, Lin Yu. Research progress of the production of the $\mathrm{LaAl}_{11} \mathrm{O}_{18}$ catalyst. J Industrial Catalysis 2006, 13(11): 62-65.

[2] Zhanlin $\mathrm{Xu}$, Lina Zhao, Liang Wang. Research progress of the New functional materials composite oxide of $\mathrm{LaAl}_{11} \mathrm{O}_{18}$. J Functional Materials 2006, 2(37): 178-184.

[3] Feng Qi, Zi-shuan Fan, Dongbai Sun. Production of the new thermal barrier coating materials of $\mathrm{LaAl}_{11} \mathrm{O}_{18}$ spray powder. J Materials Engineering 2006, 7: 14-18.

[4] Meisheng Cui, Liangshi Wang, Yongke Hou. The production of the $\mathrm{LaAl}_{11} \mathrm{O}_{18}$ catalyst and the Characterization of the Catalytic Performance of Methane Combustion. J Chinese Journal of Rare Earths 2007, 25(2):178-182.

[5] Peizhong Li, Min Xie, Ming Zhao. Preparation and characterization of the thermal barrier coating materials of $\mathrm{LaAl}_{11} \mathrm{O}_{18}$. J 2010, 31(1): 26-29.

[6] Yanqing Zhai, Yongdan Li, Ming Meng. Effect of the La, Ba on the structure and character of the high temperature catalyst $\mathrm{LaAl}_{11} \mathrm{O}_{18}$. J Applied Chemistry 2005, 22(3): 320-325.

[7] Jiandong Zheng, Jinsong Hou, Xiutao Ge. Effect of the calcinated temperature on $\mathrm{LaAl}_{11} \mathrm{O}_{18}$ catalyst. J Applied Chemistry 2010， 39(2): 198-200.

[8] Xiebin Zhu, Xuechen Duan. Effect of $\mathrm{pH}$ value on the preparation Process of Nano ITO powder produced by chemical co-precipitation method. J Rare metal and hard alloy 2006, 34(2): 8-11.

[9] Wei Y, Tang Z L, Zhang Z T. Preparation of $8 \mathrm{~mol} \%$ yttria-stabilized zirconia by an oilflotation-assisted chemical coprecipitation route [J]. Materials Letters, 2002, 57: 502-506. 